\title{
Innovation and its Importance for Contemporary Professional Life
}

\author{
(A conceptual paper with interpretation of ideas based on a close study of the existing works in the domain) \\ Dr. M.S.Bhat ${ }^{1}$, Harivardhagini $\mathrm{S}^{2}$ \\ ${ }^{1,2}$ Deptartment of EIE, CVR College of Engineering, Hyderabad, India \\ Email: bhatms@rediffmail.com, arvind.biju@gmail.com
}

\begin{abstract}
The paper provides an interpretation of work already done in the area of innovation by defining the four dimensions of innovation and their importance. The need for innovation and the kinds of innovation is discussed. The way the culture of innovations can be fostered and the importance of innovation in contemporary life is brought out clearly towards the end of the article
\end{abstract}

Index terms-- Dimensions of innovation, conceptual fluidity, conceptual flexibility, complexity orientation, Fostering culture of innovation, contemporary life and innovation.

\section{INTRODUCTION}

Innovation is a word which strikes a pleasant and familiar chord in every one of us. For that matter, who does not like to be known as innovative person? All of us cherish this word and would willingly do our best to have this wonderful and even enviable quality of human being, becoming part of our persona. Indeed, innovation is essential for society to remain vibrant and sustain. Be it in the realm of individual life or even for a community and society, constant innovation is the life breadth. Without innovation, the society would be condemned to remain stagnant and cannot ever progress. Even when a country is not endowed with natural resources, it can quite simply, more than overcome this handicap, with collective innovative mind of its human resources and become an economic superpower, as has been amply proved by the example of Japan-- which rose like Phoenix from ashes after being completely defeated and humiliated at the end of Second World War. To an extent, so also was the case of Germany. Even when all the fortunes desert a nation leaving it in shambles, it has been proven that one can rebuild afresh everything quickly, if and only if, nation's people are resilient, resourceful and more than anything else innovative!

What is innovation? How is it different from invention? Innovation is basically a process of looking for constant improvement in any thing we do. It is a quality of mind which looks for areas where one can better others; or if one is already the best in the sphere, the desire to better oneself even further. Invention on the other hand impliesdiscovery-- usually of a product or process for the first time. Needless to say that Invention is just an end by itself, whereas innovation is the means of achieving this particular end. Not all innovations need to result in invention. For the outcome of inventions tend to be discrete rather than continuous to an observer making the gap between one invention and the other too far and infrequent. However, innovation is essentially a state of mind and is the basic character of human being to improve upon something existing. Innovation is more of journey than arriving at a particular destination. And, as such, innovation as a quality is more enduring and beneficial to human kind than any other entity.

\section{DIMENSIONS OF INNOVATION}

Innovation, thus, is a state of mind which draws heavily from the fount of creativity. Innovation is founded on creativity and creativity implies thinking our-of- box. Creativity has four clear components or ingredients according to Rastogi R.N. (1996). They are conceptual fluency, conceptual flexibility, originality and complexity orientation. Rather than viewing them as components which together constitute the 'creativity', these can be viewed more appropriately, as the basic dimensions defining creativity. Though, it may be difficult to come up with exact measures of each of these dimensions and test the relevance of these dimensions empirically using proven statistical techniques, it can be logically argued that each of the four dimensions described above are by and large, mutually independent, and are vital for creativity. And creativity encompasses these basic elements, and is significant only when each of the elements is present in adequate measures. This point would indeed be clear, when one explores, what these four terms imply, and exactly what they mean in the context of creativity.

\section{CONCEPTUAL FLUENCY}

Conceptual fluency refers to the level of mastery over the subject of an individual. The extent of knowledge must be to such a level, enabling the person to grasp fully all the aspects of the subject comprehensively and explain it to others neatly in clear terms in such a way that others feel fully satisfied, and for all practical purposes, consider the person as immediate and convenient source of knowledge on the subject. It is indeed quite simple to understand its importance as a dimension of creativity, as one cannot be innovative or creative without a strong streak of basic knowledge in the field where one intends to contribute. The creativity requires application of specific knowledge and the knowledge should be forthcoming unhindered and freely. For this, one needs to acquire a strong foundation of knowledge either theoritical or experiential on very firm footing. The stronger the foundation, better are the chances of creative ideas to emerge. If we examine the genesis of all earlier innovative endeavors, it would be 
apparent that the new innovation is mostly a continuum of all the earlier innovation. Even where it is, sort of breakthrough, and clear break from the past, on closer examination, it would be seen that the present innovation would have made use of some or other element of the earlier work on this domain. If one cannot leverage the existing knowledge for future advancement, it is clear that very little new knowledge can be added ab initio or a fresh one without any link to the available fund of knowledge. The ability to move cognitively along one's sphere of chosen area effortlessly and freely is thus termed as conceptual fluency. Conceptual fluency is thus the basic and essential dimension of creativity. If one has difficulty in navigating or freely traversing in one's own chosen area of specialty, it is clear that very little creativity can forth come from the individual concerned. The conceptual fluency therefore, should logically enable a person to generate large number of solution for a given problem based on one's expertise in the field. In the chosen area of knowledge, the conceptual fluency would eventually enable the mind to become very fertile due to complete internalization of the concepts already imbibed, and seeds of ideas get germinated and sprouted, no sooner the problem is encountered.

\section{CONCEPTUAL FleXibility}

Conceptual flexibility refers to the cognitive ability of the person to move seamlessly from one perspective to another or from one frame of reference to another. If a person has fairly good understanding and grasp of different spheres of knowledge the process would seem quite natural. The ability to cut loose from rigid boundary of one domain of knowledge and move to a different domain can be indeed a very valuable quality of a person, enabling him to synthesize something totally different and new. It is apparent that analogies abound this world as one can discern patterns in nature. And patterns do have similarities. It is always fascinating to learn that a geartrain in the domain of mechanical engineering is no different from transformers of electrical technology, a mechanical spring is analogous to inductor of electrical engineering and a flywheel is mechanical equivalent of capacitor. The analogy is not just confined to components; it extends to sub-systems and even system too. Most of the branches of social sciences extensively draw analogy from physical sciences to buttress their points. For the world is full of symmetry and pattern; and yet each one of us is singularly different to the extent of being unique! The conceptual flexibility, therefore, is a quality which enables one to liberally go for transfusion of ideas from one sphere of knowledge to another with singular purpose and yet respect the uniqueness of each sphere of knowledge. Without the development and use of analogies, and analogical tools from the 'foreign' or external disciplines, one cannot hope to develop one's own field of interest. The insight acquired through analogies is often spectacular and quite deep. The phenomenon of conceptual flexibility is akin to an individual moving freely and effortlessly across the international borders, fully respecting the sovereignty and sanctity of the international borders of the nations of the world. Conceptual flexibility is thus a quality, enabling a person to see a problem from many different angles or perspectives and then arriving an integrated picture with all its subtle nuances. In this context, it is worth recalling the saying of Oliver Williams. According to him, 'Many ideas grow better through symbiosis or process of transplantation from one mind into another mind, than leaving it to nurture just in the place where they sprang up'.

\section{ORIGINALITY}

According to the definition by Rastogi R.N. (1996), originality is the basic acumen of human being to produce, unusual, unconventional, novel, atypical and audacious answers to questions, least expected responses to problems, and unique unorthodox refreshing interpretation of issues, situations and events. Originality entails a person to avoid following the beaten track and always blaze a completely new trail. Even if the path is completely un-trodden, unpaved and difficult to negotiate and traverse, the quality of originality would entail one to choose a path less walked. A serendipitous mind is the most significant aspect of the person who is always original. The person with characteristics of originality would often discount the common approach followed by most of us as just one of the many possible approaches to the problem and never consider it as the best solution. While original thinking is to a great extent, is an innate quality of a person, with constant training and effort, it is always possible that one can develop this distinct quality with considerable effort and perseverance.

\section{COMPLEXITY ORIENTATION}

Going by the definition of Rastogi R.N.(1996), complexity orientation is the distinct characteristic of the person to confront, challenge and find meaning, in complex and ambiguous situations, and to enjoy the very act of analyzing, integrating, clarifying and resolving them. As a matter of fact, this is one of the essential attributes of any leader. A leader is one who can find simple solution for complex problems. Order out of chaos or reducing complexity to comprehensible simple things is indeed the way one would prefer things than letting simple thing go complex or letting orderly things eventually move into chaos. According to Machiavelli, the opportunities offered by a good crisis are enormous and very valuable, and one should never let go the opportunity thrown up. The leader recognizes the fact that the higher the complexity of the situation, more pressing is the need for immediate and qualitative solution with the possibility of a solution stretching the human potential to its limit. On the other hand, a simple situation with a simple problem can be routinely solved and it cannot be exciting to people who look for creative adventures. Complexity orientation is to a great extent contingent to the level of one's intellectual advancement and growth in emotional maturity. Whereas, the lesser beings or mortals are comfortable with simple situations, and would prefer to countenance simple problems, and are never at ease with 
situations making them leave their comfort zones, and often balk at complex situations, a person of complexity orientation would exactly love the opposite situation. But for this category of people, who constantly look for challenges and complexities in life, the society and social structure would be stagnant and there would not have been improvement and progress at all.

\section{NEED FOR INNOVATION}

The need for innovation arises from the fact that most of the human beings, i.e. even those working in business organizations which are well structured and societal organization which may not be so well structured, are overwhelmed by activities which are purely transactional in nature, and often they do not realize this fact. The everyday routine transactional works are so pressing and so large in volume, so much so, that an average individual thinks and sincerely believes that-- that is real work-whole of work and nothing but this type of slogging work is that enables organization sustain and even progress. The truth cannot be farther from this notion. There could be occasions when one faces some kind of challenges initially in work, and this may be often, purely transactional in nature, meaning thereby a job requiring a bit of tweaking of the existing arrangement. And the employee gets over this challenging situation mostly by following the suggestions and the advice of one's peers. On a relatively fewer occasions, when an employee comes up with an innovative solution for the problems, for a while, the employee thinks that it is unusual response and momentarily lauds himself. Thereafter, the employee tends to rest on his laurel rather than continue with the quest for innovation for its own sake. In a situation where the external factors are quite stable and conducive, the employees, for most part of their life, can continue with this state of existence and lead a contented life. Only when the problems are to be solved from the perspective of fundamental change, arising out of complex dynamics coming into play, or disturbance or even disruptions in external conditions, there is a need for total transformation of the process, large scope for innovation suddenly appears on the horizon. With the boundaries of knowledge and technology are ever widening and expanding, even to remain in the same place in the competitive world one needs to innovate. The world does not change due to people who ordinarily comply without questioning, and prefer to go by precedence without thinking originally. When a person refuses to go by the beaten track and questions the existing procedures critically, people around him sit up and take notice of the person and are forced to examine the situations. And these are the situations that can cause transformational changes and, only transformational attitude can be the driving force for innovative thinking and progress. If the need for innovation is indeed an undisputed and unvarnished truth, the act of innovation is fully dependent on transformational thinking and action. Invariably the transformational thinking would imply, pushing the outer envelope of one's endeavor or domain of activity, farther and farther. And without innovation, one is doomed to stagnate, and ultimately would be wiped out entirely from the scene. And hence for progress and moving ahead, whether from the perspective of society or business, innovation is an imperative and a desideratum.

\section{KINDS OF INNOVATION}

Based on the value addition, Elaine Dundon (2007) famously categorized innovations into three types, namely, efficiency innovation, evolutionary innovation and revolutionary innovation. It must be noted that the division of innovation into three groups can never be presumed to be watertight and compact. Rather, it can only be said that the categorization based on value addition of innovation is a kind of continuum on the innovation value addition scale. Those innovations contributing in a significant way to the present state of the art technology is rightly termed as revolutionary --as it is a kind of drastic departure from the present line of thinking - which disturbs and even dislocates the existing state of affairs. Evolutionary innovation, on the other hand, is a process of building and leveraging on the present, without breaking away completely from the past. In a way, it is an approach of incremental work on evolutionary basis and the whole process is aimed at contributing moderate amount of value addition. Efficiency revolution on the other is purely incremental in nature and is aimed at cutting cost, improving efficiency, improving quality and providing a better appeal to customers and so on. As the effort is to achieve better efficiency and get more mileage from the existing situation, the whole approach is one of working around the present technology. It is therefore obvious that, both efficiency and evolutionary innovation operate, by and large, within the existing frame work of innovation without total break or complete rupture from the existing state and appears to be a kind of seamless progress along a continuous line. An entrepreneur, therefore, ideally would be required to straddle all the three types of innovation, moving from one mode to another seamlessly with ease according to the need and exigency of the situation. This particular attribute would help a great deal the entrepreneur in carrying out his plan of growth in the short, medium and long time horizons and take the venture too far.

\section{Fostering Culture Of InNOVAtion}

In view of the significance and importance of innovation, at all levels of entrepreneurship, business, society and government, it is necessary to assiduously and carefully cultivate, nurture and develop innovation both as a trait, credo and way of life. It must be remembered that innovation is not just an inborn quality but can also be nurtured and developed through systematic training by following established and proven techniques. There has been considerable work in this area by Edward De Bono(1993). While the exact techniques can be gleaned or studies from the literature, the underlying feature of all these techniques rests on the fact that one need to employ the full potential of divergent thinking-- apart from the conventional convergent thinking. The convergent thinking has always been the focus of development at the 
childhood, schooling, secondary and tertiary education under the present system in majority of the cases. The process of convergent thinking revolves around clearly identifiable steps of problem identification and subsequent definition, diagnosis of the situation and the problem, and systematic analysis followed by prognosis, and synthesis providing solution to the problem. On the other hand, divergent thinking generally follows the process of identification of the problem on a tentative basis, searching solution starting with most familiar and then moving to complex ones, shifting one's perspective and frame work quite widely, all the time. To put it simply while convergent thinking is movement along closing spiral, the divergent thinking is movement along ever widening spiral. The search for solution is accomplished through a process of divergent movement along the locus of expanding concentric circles, moving from the simple obvious looking solution at the centre to more non apparent, novel and innovative solutions at the periphery. In the process, one might use analogies from other realms of study or knowledge. Indeed the ability to bring into play large number of diversity or variety or perspectives, in terms of ideas, imagination and creative outcome can be of great use. The right hemisphere of the human brain is associated or credited with intuitive, imaginative and creative thinking process and traditionally, in our quest for imparting reasoning, logical thinking-- which are all part of convergent thinking,-- not much attention is paid to the development of imagination and creative endeavor in childhood. It is therefore imperative, that one concentrates on development of this aspect right from the childhood by engaging in stimulating creative activities like singing, painting and story telling, even as one concentrates on inductive and deductive logical thinking aspects of one's development. The ability to entertain and examine all ideas without necessarily accepting them is one of the important requirements for divergent thinking. As Mahatma Gandhi said, "I do not want my house to be walked in on all sides and my windows to be stuffed. I want the cultures of all lands to be blown about my house as freely as possible. But I refuse to be blown off my feet by any."

\section{THE IMPORTANCE OF INNOVATION FOR CONTEMPORARY LIFE}

The astonishing progress of mankind in this world has been mainly due to ability of the human being to think and innovate constantly. The locus and trajectory of the path of progress is solely determined by the quantum and quality of springs of innovative-thinking in a society, organization or establishment. We have witnessed societies and organizations prospering when the emphasis and stress is on promotion of innovative thinking and innovation at every level. On the other hand, we have equally large number of examples, of organizations and societies languishing, remaining undeveloped or even decaying when they stopped innovating. The process of innovation is not necessarily confined to technology, arts, sports, literature, science, etc. and covers every aspect of human endeavor. Indeed the boundaries of innovation are never fixed and keep expanding with time. Innovation is ever welcome and is essential in every sphere of the individual growth namely, physical, sensory, emotional, mental, intellectual and ultimately even in spiritual. Innovation in every sphere, therefore, is the process of human being seeking and attaining a higher level of consciousness than hitherto achieved and this process is never complete. For the true measure of human potential is still unknown, or at best known very little and every innovation is thus a step towards knowing our own true self and its depth.

While the importance of creativity and innovation in the field of technology and business seems very obvious and is readily accepted as the key determinant for success at individual and organizational level, what is not so obvious is, the significance and importance of innovation in every walk of life or avocation and its all pervasive and ubiquitous nature. Innovation, thus, should become an ingrained character of an individual. The life would then become a matter of series of interesting challenges to be overcome with interest and passion by the individual. The joy and fulfillment in identifying and confronting a problem and solving to the satisfaction of everyone, is indeed a true inner joy, which only human being with higher level of consciousness can indulge in and appreciate. Thus, , being totally absorbed and indulging in exercises in creativity and innovation for its own sake, is rewarding to human kind. For innovation implies constant learning and, without learning society stagnates and putrefies. As John Naisbitt has remarked, "In a world that is constantly changing, there is no one subject or set of subjects that will serve you for the foreseeable future, let alone for the rest of your life. The most important skill to acquire now is learning how to learn." And that indeed is the key to inculcate innovative spirit in individual, organization and society.

\section{Conclusions}

Technology is the cutting edge of growth and progress of society. The advancement of society to a large extent, hinges on the ability of the society to leverage its technology and intellectual capital. If one looks at the pace at which technology is growing, it is, at once, evident that the innovation is the engine which propels continuously the advancement of technology along its high speed trajectory. A teacher who does not grasp the significance of innovation in technological advancement and its role in engineering education is obviously at a great disadvantage. Just as technology calls for constant innovation, the teaching of technology or engineering education calls for sustained and constant innovation. Indeed viewed from this angle, the innovation in engineering education is more challenging than innovation in technology. What else can be a better place to learn innovating in engineering, other than the engineering college where the student cuts his teeth and to which students come with great hope? The culture of innovation should therefore rightly start at colleges with engineering education taking the lead and one need to pay greater attention to this important aspect. 


\section{REFERENCES}

[1] De Bono, E,(1993) Parallel Thinking, Viking London

[2] De Bono, E, Sur/petitions, Harper Collins,New Delhi 1993

[3] Elain Dundon,(2007) The Seeds of Innovation-Cultivating the Synergy that Fosters New Ideas. Prentice Hall of India Private., Ltd., New Delhi 